\title{
RECENT ADVANCES IN PHYSIOTHERAPEUTICAL METHODS
}

\author{
By DR. WILLIAM BEAUMONT
}

Director of Physical Medicine, Westminster Hospital.

\section{INTRODUCTION.}

\begin{abstract}
ATHOUGH Physical Medicine is now a widely recognised form of treatment, it cannot be said that any dramatic or revolutionary methods have been evolved to account for this changed attitude. Before the War, Physiotherapy was the cinderella of therapeutic measures, and the Physiotherapy Department was very largely a dumping ground for the unwanted but tenacious outpatient. True there have been marked advances in diagnostic methods, but these are the province of the Physical Medicine Specialists, themselves one of the most recent. additions to the Consultants and Specialists Group.
\end{abstract}

Advancement has been made in two directions. Firstly in the design of apparatus which is now based on the electronic valve. This modern development has displaced the old and wasteful spark-gap circuit for diathermy and the induction coil, metal rectifiers, etc., circuits used for producing the faradic, sinusoidal and galvanic currents. Secondly in regard to treatment. Basic physiotherapeutic technique has been adapted and employed in many spheres of medicine and surgery, both old and new, so that fresh fields of interest and usefulness have been opened up for the physiotherapists. Thoracic surgery is one of the most spectacular examples, but the wide-spread use of pre- and post-operative treatment in general surgery, the employment of muscle relaxation exercises for psychiatric cases $(\operatorname{Ref} .1$.$) , ante and post natal exercises (Ref. 2.),$ the treatment of respiratory conditions (Ref. 3.), the recognised position of the physiotherapist in the plastic surgery team (Ref. 4), are all indications of the modern trend of expansion in the use of physical methods.

The writer has chosen two examples to illustrate recent advances in methods of treatment. As this article is written specially for the physiotherapist, details of technique will be given as fully as possible.

\section{THE SLOW HEALING WOUND.}

One of the more recent advances, but perhaps least known or rarely used physiotherapeutic methods, is the one applied to tissue repair. In cases of malignant neoplasm far reaching excision of tissue is the practice. In these and in some cases of non-malignancy, incisions, often quite deep through unaffected tissue, have to be made. Most of them heal by first intention. Some, however, break down and healing is arrested.

It is therefore of interest to all to find a method, or methods, which will initiate or stimulate tissue repair. At one time it was thought that healing was arrested by bacterial invasion, and chemical agents were used to sterilise the wound, but these were found to destroy not only invading organism but healthy tissue cells, and so delayed healing. The discovery of the new antibiotics was hailed as the solution to the problem, but time has shown that these are not infallible. New avenues of research, therefore, are being explored in the hope of finding a means of stimulating tissue repair activities, with the result that an old and almost discarded physiotherapeutic method is being revived. Before describing this it will not be considered out of place, it is hoped, to outline the normal process of tissue repair.

\section{Mechanism of Granulation:}

The various stages by which granulation tissue is formed need only be summarised very briefly. The area is flooded with a rich protein exudate which ultimately coagulates. At first this forms a matrix for the white cells, which migrate through the vessel walls, and for the connective tissue network formed by the fibroblasts. Coincident with this the neighbouring capillaries throw out projecting buds. These elongate, join with other similar buds, and form loops of new capillaries which become entangled in the connective tissue network. The exudate coagulates, cementing the whole together, and a new kind of tissue is formed, highly vascular and easily damaged. This fills in the crevices and builds a structure filling in the entire cavity. Ultimately the connective tissue network contracts, epithelial islets appear on the surface, or push out from the edges of the skin, and complete the process of repair.

What initiates this complex tissue activity, and what means are at our disposal for bringing this about, is based largely on hypothesis. In 1858 Virchow pointed out that healing was the result of direct stimulation of cells by trauma. After a long interval the theory was revived. Wiesner suggested that the traumatic effect was an indirect one the injured cells releasing substances which stimulated normal cells to proliferate. Eventually the nature of this substance was determined and believed to be that of a 'hormone', and subsequently identified as tissue promoting. The present view is that trauma is the primary causative agent, and the repair process results not from destruction, bur from partial damage of the cellular elements. Thus, for example, the surgeon's knife destroys but leaves damaged tissues in close proximity to the incision, and from these the mechanism of tissue repair is initiated thus presenting a possible explanation of healing by first intention.

Research in relation to the effects of the shorter ultra violet rays, given in non-destructive doses, has shown that they damage but do not destroy cellular elements The most effective wave length is believed to be in the region of 2,000 A.U. Thus it would appear that radiations of this wave length operate as a traumatising agent (Ref. 5.) liberating a hormone-like substance within the wound which is capable of stimulating, or re-starting, the process of granulation in cases where it is inhibited or even ceased to function. This hormone theory possibly does not fully explain the entire process of healing. The air ionising properties of ultra violet radiations, the result of exciting molecular activity are certainly contributory factors. The smell of ozone in the ultra violet radiation room is well known, and is attributed to air ionisation (Ref. 6.). This ozone can be detected emanating from the skin of some persons after ultra violet irradiation and also in a wound after irradiation. This may account for the antibiotic effect. Increased oxygen production in the tissues has been attributed to this ionising action of ultra violet radiation, resulting in the acceleration of respiratory exchange within the tissue cell, which it has been demonstrated also has a beneficial effect on healing. Lastly, one other result of research has shown that when cells are exposed to ionising radiations, variation in the cell nucleus takes place, resulting in changes in cell characteristics (Ref. 7.). It may well 
be that herein lies a germ of an idea which accounts for appearance of the fibroblasts and the vascular budding are essential forebears of granualtion tissue. As has which areen stated the full explanation of tissue healing already heen stathothetical state and the above theories is as yet in a a probability and not as an etabhed fact. are presented as a can be little doubt that the clinical results However, there a rational and not purely empirical basis. observed hav

Technique:

This must follow a definite plan, formulated on the nature, the site and the extent of the wound, and whether it he a simple or complex wound which has to be dealt with. The plan must take into consideration also the need with. The plapentary treatment and the method for preirradiation cleaning and post-irradiation dressing.

\section{Supplementary Treatments:}

Movement of surrounding tissue and joints is essential to maintain high metabolic activity, and active exercise to me part does much to improve circulation and lymphatic drainage. It is often necessary to explain to the patient that movement will help in his recovery and so counter that movement will immobilisation. Where the patient's the tendencition does not permit of voluntary movement, neuro-muscular stimulation can be instituted to minimise muscle atrophy and joint stiffness and improve circulation.

Infra red will relieve the pain and stiffness following enforced immobilisation and so make movement easier. It also assists in increasing local tissue metabolism. The part played by infra red irradiation must not be underestimated, bearing in mind that any effective ultra violet radiation generator also emits a large percentage of infra red (Ref. 8.). General ultra violet irradiation can benefit materially the patient who is psychologically and constitutionally at a low level.

\section{Pre-Irradiation Cleaning:}

This is best done with a few drops of neat peroxide, followed by warm saline irrigation. Peroxide because it liberates oxygen, and warm saline because it closely resembles tissue fluid. Excess of residual moisture should not be dealt with by mopping with wool or gauze as particles are left behind and the delicate new granulation tissue is damaged. Drying is best done by exposure to infra red and can be accomplished in 5 or 10 mins.

\section{Post-Irradiation Dressing:}

To prevent the dressing sticking, gauze impregnated with halibut oil, or cod liver oil, is laid over the wound, but not pushed into it. Wool and bandages can be applied over this. Glucose powder can be used for dry superficial areas.

\section{Irradiation Technique:}

The Kromayer lamp, with suitable quartz applicators, and the disc type non-luminous infra red generator, are the apparatus of choice for all types of slow healing wounds.

\section{An uncomplicated wound:}

By this is meant one without sloughs, a sinus, or deep cavity. The site chosen for the initial irradiation should be damaged, but not destroyed, tissue as evidenced by existing granulation and not in close proximity to bone structure. If such tissue forms the floor of the wound, so much the better. A quartz electrode is necessary for deep wounds, and the plated type is best, except for cavity work, to be dealt with later. This localises irradiation and makes for better dosage control. It should be long enough to reach to within $\frac{1}{4}$ of an inch of the chosen area. In shallow wounds the applicator holder alone can be used.

Dosage:

Bearing in mind that the short ultra violet radiations are the most effective, distance is important, not because of the Inverse Square Law, but because of air absorption and the need for the maximum intensity of rays falling on the area at right angles. (Cosine Law).
Distance therefore must be as near as possible without contact, varying the distance to the irregularities of tissue level. Even momentary contact will result in some of the young granulation sticking to the electrode face. Whether the area be large or small, the best method is to keep the electrode moving all the time. Concentration on one spot can destroy the vascular buds in a short space of time.

Time:

The effective time is much longer than that required for skin lesions, and can be estimated approximately at $1-3$ mins. for each inch square, providing the applicator is kept moving. Judgement must be exercised in estimating time as there is no mathematical precision in the growth of granulation tissue. If the response is not good, increase the time, if the granulations lose their red glistening appearance, reduce the time as this is an indication of cellular destruction. An ineffective dose, however, will not give good results.

Complex Wounds:

This term is used to describe a wound in which there is sloughing, a sinus, or a cavity, or all three. In dealing with such a wound it is the more or less healthy tissue to which irradiation must be directed to produce the cell trauma already referred to.

Sloughs:

Very large and thick patches of sloughing must be removed surgically. Smaller areas are best attacked indirectly. Snip away any loose edges and then irradiate the marginal tissue so exposed, after cleaning with peroxide and saline. Adopt the standard unit dose and use the electrode which is most suitable, the plated curved one usually is required. As the slough recedes, attack the new margin exposed.

A Sinus:

This also must be attacked separately. Cleaning may have to be done before treatment for several days at first. Time must be allowed for drying by absorption and infra red, not 'mopping up'. Also the tissue should be sensitised to counteract the inhibiting effects of the cleaning. A $1 / 1000$ solution of Eosin, applied with a pipette will be found effective. Only a few drops are necessary, and allow time for the sensitiser to operate. To prevent surface closing the insertion and withdrawal of the electrode should do all that is required, but as the opening gets smaller, gauze wedged in between the edges for not more than one quarter of the total depth may have to be used. A small sinus, forming part of a large wound, will usually close in without special attention, apparently taking part in the general tissue repair. The standard nasal applicator has many disadvantages, chief of which is very limited radiation emission. A modification, plated and of greater diameter, has been designed, and this gives increased radiation and can be used to stretch the opening.

The larger sinus, which readily admits of a standard size electrode, is dealt with in the following manner after the cleaning process. Both depth and direction must be determined before treatment by using a suitable electrode as a probe. The working depth is $\frac{1}{2}$ an inch less than that obtained. The 'bubble' electrode is most useful and the time for the first three or four treatments is ten minutes. As the sinus fills in, reduce the distance and the time. Care must be exercised as regards depth. If obstruction is felt at any time during treatment, withdraw the applicator $\frac{1}{2}$ an inch. When granulation tissue becomes visible, discontinue insertion and follow the lines laid down for a simple wound.

\section{A large, deep cavity:}

This also requires individual attention. Cleaning up before treatment is a problem. It is usually done by allowing the patient to have a bath, otherwise by daily irrigation of the cavity itself with warm saline. Non-.luminous infra red is given until the cavity is dry and warm. Again use the sensitiser as previously described, do not flood the cavity, and dip the electrode into a pool of sensitiser. 


\section{Electrode:}

These cavities have a deceptively large surface area, quite irregular in depth. Scattered radiation is therefore necessary, and this is obtained from the bubble electrode.

Intensity of radiation is reduced and therefore $15 \mathrm{mins}$. may be necessary if the cavity is large, but 10 mins. is sufficient for the size of the cavity usually seen.

\section{Stimulation of epithelium:}

When the granulation is within $\frac{1}{2}$ an inch of the surrounding skin level, attention should be paid to the edges. These may need to be traumatised by scraping away the stratum corneum which has formed a protective covering. No harm is done if this results in slight bleeding, in fact it is a good thing, it can be arrested easily and the edges can then be irradiated. Using a plated applicator and adopting the labile method, a quarter of the unit dose, as regards time, can be employed. The greatest care is necessary not to encroach more than $\frac{1}{2}$ an inch on normal skin. A definite perceptible degree erythema is all that is required. Wherever budding of the epithelial margins or epithelial islets appear, encourage and carefully protect these points. Often it is possible, at this stage, to split a large superficial granulating area into one or more sections by concentrating on this epithelial activity. This lessens the time for complete coverage and makes the work less tedious.

\section{Pressure sores:}

No special reference will be made to these as the technique of treating them is the same as outlinet for the uncomplicated wound.

\section{PERIPHERAL CIRCULATORY DISEASE.}

This is a generic term applied to a group cf conditions with differences both as regards pathology, etiology and cause, but all may have a similar clinical syndrome and termination. They all have this in common, there is a disturbance of the peripheral circulation, terminating in thrombosis and in some cases ultimate pulmonary or cerebral embolism. The technique described can be applied equally satisfactorily to all of them, with such minor modifications as are dictated by the individual case and common sense.

\section{Thrombo-Angiitis Obliterans:}

Better known to all physiotherapists as Buergers Disease. The symptom syndrome comprises intermittent claudication, i.e. cramp-like pain coming on after exercise, particularly walking, as the lower limbs are most often affected, cyanosis when the limb is hanging down and paleness when elevated, reduced or absent pulsation normally felt where the dorsalis pedis branch of the anterior tibial artery is superficial. In addition there are skin changes, at first lowered local temperatures, 'chilblains', later developing into 'ulceration'. The onset, more often than not, goes unnoticed until it becomes an inflammatory lesion of a vessel; i.e. phlebitis which leads to thrombosis, but embolism is not associated with this condition. The cause is unknown.

Thrombo-embolic Disease:

This may be either a primary medical condition or secondary to surgical procedure. Like the previous condition the onset is characterised by phlebitis, thrombosis supervening at a later stage. Unlike Buergers the risk of a pulmonary or even cerebral embolism is a definite one, as the name denotes. The early stage, i.e. thrombophlebitis, is when physiotherapy is most useful, as little can be done in the acute phlebitic stage, and when embolism has occurred it is not uncommonly fatal if it be a pulmonary embolus. The incidence of thrombo-embolic disease is on the increase and the cause, while not definitely known in the non-surgical type, is attributed to the present way of life and the growing increase in the older age group.

The post-operative condition is attributed to advances in surgical procedure made possible by modern methods of anaesthesia, long periods of immobilisation and interference with circulation of the large vessels and the heart itself. The increasing use of intravenous injections and the need for blood transfusion are believed to effect blood changes both in regard to volume and coagulability and so predispose to lowered blood pressure and inflammatory vessel changes.

From this brief outline of the symptoms and the etiological factors it can be gathered that the problem that presents itself is whether physical methods can make any contribution, either directly or indirectly, to the reversal of a pathological state characterised by circulatory stasis, whether due to disease of the vessels or mechanical interference. Let us consider the physiological response of the various physical methods in general briefly and two in particular.

\section{Treatment:}

In the present state of our knowledge treatment must be directed towards slowing coagulation rate and/or compensation for loss of natural contraction and dilation of the vessels. The use of anticoagulants, e.g. Heparin, is almost a routine where thrombosis is imminent or established. This is effective where the vessels are able to perform their natural function, and when the blood has acquired its normal or nearly normal fluid state, in which case physical methods assist the physiological action of the vessels.

The alternation of rest and functional activity of the vessels is provided by alteration in their calibre, the result of autonomic system action and the independant action of voluntary muscle. The object of the surgical procedure of sympathectomy is to interrupt the vaso-constrictor mechanisin under the control of the autonomic system. Before this operation was introduced the only method available for varying the calibre of the vessels was by means of external pressure. Before discussing this aspect of treatment it is well to review the orthodox physical methods of massage, heat and exercise in the light of our present day knowledge.

\section{Massage:}

This is definitely contra-indicated in the acute inflammatory stage of phlebitis and the pre-thrombotic state. Opinions differ as regards its use when thrombosis is established. One reason is based on the assumption that it might result in breaking up the thrombus and so initiate an embolus, the other is that massage can have no effect one way or the other on thrombus formation.

\section{Heat:}

It is a common frequent clinical observation that the application of heat to the extremities of patients suffering from organic arterial disease increases pain and especially where gangrene occurs, causes extension of tissue necrosis. It has been demonstrated that local application of heat produces deficiency of circulation by increasing local tissue metabolism. Damaged vessels are unable to supply an adequate blood supply and local heat increases this disproportion. Heat therefore is contra-indicated in obliterative vascular diseases and may accelerate the onset of ulcerative process. In the inflammatory stage of phlebitis it can be beneficial if the non-luminous source of infra-red irradiation is used (Ref. 8.).

\section{Active Exercises:}

There is universal agreement that active exercises improve circulation and the well known system of Buerger's exercises, if accurately carried out, is most valuable in all forms of peripheral circulatory disease. Patients should be made to understand that it is imperative for them to memorise these exercises and practise them at home or at work as often as possible during the day as well as night and morning, and Ward patients should be encouraged to practise for tweaty minu'es every two hours. It cannot be too often repeated that the role of the physiotherapist is that of a teacher and her supervision periods, whether in the ward or the department, are primarily for perfecting technique and correcting faults. This system of active 
exercises is of special importance in post operative routine and in association with the use of coagulants.

\section{Environment Pressure:}

This is the name given to a system of treatment designed to stimulate an adequate circulation through the collateral arterial pathways by rythmically decreasing and increasing air pressure about an affected extremity. A specially desiair pressaratus called the Pavaex Unit is used. The name gned aived from PAssive VAscular EXercises. Briefly, the limb is placed in a Pyrex glass boot fitted with an inlet nipple to which is attached a rubber tube connected at the nipple end to an apparatus, the operative feature of which is a motor-driven pump with rotary or sliding, pressure operated, valve. By this device the limb can be subjected to controlled alternation of the environmental pressure within the boot. The assumption on which this method is based is that such alternating pressures external to the limb will result in alternating occlusion and dilation of the vessels. The Pavex apparatus is rarely seen in a modernised department, and the method is rapidly disappearing and is being replaced by the following.

\section{MUSCLE STIMULATION.}

The method to be described may be considered a recent innovation, or at least an adaptation of an old method put to a new use. In nerve lesions or disuse muscle wasting the application of a faradic current to a motor point is used for stimulation of a muscle or small muscle group and it is important not to involve unaffected and particularly opposing muscles. The object of using this method in the treatment of peripheral circulatory disease is to obtain contraction and relaxation, not of a small group but the whole muscle mass in which affected vessels are embedded, irrespective of the primary function of those muscles. The hypothesis on which this method is based is briefly as follows:

Contraction of muscles affords effective aid to venous return and can be obtained by electrical stimulation. This has been demonstrated by experimental work on animals (Ref. 9.) and that elecrically produced contractions increase the venous outflow (Ref. 10.). From this it is deduced that by securing this electrical muscle response, the vessels will be alternately compressed and relaxed. During the occlusion stage blood flow will be arrested, and released during relaxation with increased force and volume. In this way a cycle of alternating vessel rest and activity is established.

Muscle contraction needs to be strong enough to occlude the lumen of the vessel and the timing of the release to synchronise with the pump action of the heart. As yet the results on animals have not been demonstrated, for obvious reasons, on human beings.

Technique:

Position of the patient: In order to neutralise the effect of gravity and so assist muscle action, the affected limb is elevated throughout treatment. This is best done by reversing the nomal position of a patient on a treatment couch. The feet and legs resting on a pillow are supported by the raised headpiece of the couch.

Electrodes:

These need to be flexible and thin to avoid localised pressure. Thin flexible aluminium sheet metal is suitable material to use. It is easily cut to the desired shape and size and two layers of lint can be stitched on to the metal. Metal foil backed with felt saves trouble, but does not last long. Alternatively, 'electrode jelly' can be smeared on the surface of the metal and applied directly to the skin. This obviates the unnecessary use of moist lint or felt. One electrode forms a cuff round the calf muscles embracing the main portions of the belly of the gastrocnemius, soleus, peroneus longus, anterior tibial and extensor longus digitorum, but excluding that portion of the shaft of the tibia which is usually superficial. The other electrode is placed on the sole of the foot, excluding the toes and heel. Both are fixed in position by narrow strips of elastoplast. A thin rubber bandage is then applied evenly to the entire limb from toes to knee without causing any sensation of constriction. In cases where both limbs are affected, similar electrodes are applied to each limb, those on the feet being connected by a bifurcated lead to one terminal and those on the calves are connected in the same manner to the other terminal of the current supply. Small thick, bulky pads must not be used as they cause localised pressure on the more superficial vessels.

Current:

The sinusoidal or interrupted galvanic was first used and some workers consider the latter to be the best available at present, as the pulsation rate can be timed to synchronise with the pulse rate by means of a metronome. This type of apparatus is rapidly going out of production. The modern apparatus made by British firms are electronic generators of the rectangular current pulse of controlled amplitude and duration, from which can be obtained a current of $300 \mathrm{~ms}$. at 1 pulse per sec. This is a far more pleasant current from the patient's point of view than the interrupted galvanic. The writer-is using all three types at present in the hope of assessing the therapeutic value against the various disadvantages of each.

Dosage:

This can be expressed in terms of current, duration and frequency of treatment. Current strength is of the utmost importance and there can be no rule of thumb. The essential point is the production of visible strong contractions; without these, treatment is ineffective. As regards the duration and frequency, workers in the United States who use a specially designed apparatus, with many useful refinements, give continuous treatment varying from twelve to twenty-four hours (Ref. 11.). The writer is unaware of any procedure comparable to this being used in Great Britain. The usual procedure is to give $\mathbf{3 0}$ mins. twice a day. Admittedly it is not ideal, but it is practical for in-patients if not for out-patients, for whom treatment is given daily. General observations:

The results of using muscle stimulation have been clinically satisfactory. Combined with Buergers exercises the results are better than either method alone. Used alone it is most useful in post operative cases while the patient's general condition prevents active exercises.

The two conditions dealt with have been selected because the methods advocated have given good results and either they are not known or for various reasons not used. Moreover these new spheres for work for the physiotherapist require the revival and adaptation of some of the older physiotherapeutic methods.

\section{LIST OF REFERENCES.}

1. Muscle Relaxation: G. Garmany, Actinic Press, London.

2. Natural Childbirth: H. Heardman, E. \& S. Livingstone Ltd., London.

3. Respiratory Function: R. D. TonkIN, Actinic Press, London.

4. Physical Methods in Plastic Surgery: J. P. ReIDY, Actinic Press, London.

5. Tissue Repair: Leo G. Nutini, Glasser: Medical Physics, Chicago, Illinois.

6. Ozone. Effects of Ultra violet Radiations on Cells: J. R. LoOfBourow. Growth Symposia.

7. Effects of Ultra violet Radiations on Mutation MUller \& Mackenzie. Nature, Jan. 14th, 1939.

8. Infra Red Irradiation-3rd Ed.: WM. BEAUMONT, H. K. LEWIS, London.

9. Journal of Medical Science. Oct. 1948

10. Proceedings of Experimental Biology \& Medicine, Nov. 1948

11. Archives of Physical Medicine, Jan. 1954. Thromboembolis disease. Martella \& OTHERs. Chicago, Illinois. 